\title{
METODOLOGÍA PARA EVALUAR EL PLAN TERRITORIAL DEL CANTÓN PORTOVIEJO
}

AUTORES: Blanca Victoria Cerruffo Briones ${ }^{1}$

Angela Marcelina Cerruffo Briones ${ }^{2}$

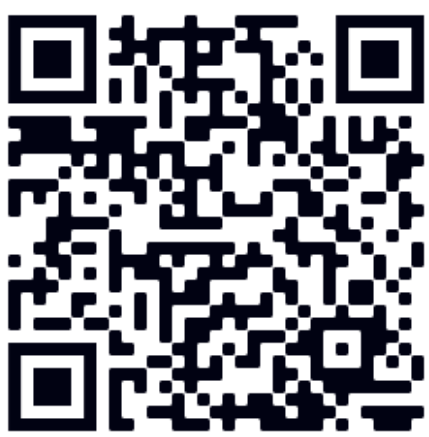

DIRECCIÓN PARA CORRESPONDENCIA:victoriacerruffo@yahoo.com

Fecha de recepción: 17/06/2019

Fecha de aceptación: $20 / 07 / 2019$

RESUMEN

El cantón Portoviejo es una ciudad de la provincia de Manabí perteneciente a la región costa del Ecuador. La población según el último censo poblacional es de 280,029 habitantes. En los últimos años ha tenido un gran avance en cuanto a urbanismo, adecuación e infraestructura de servicios básicos fruto de la planificación desarrollada por el municipio. Pero aún falta muchísimo por hacer para que Portoviejo pueda proporcionar las mejores condiciones de vida para sus pobladores. Para el efecto el municipio diseñó el Plan de desarrollo territorial que se encuentra vigente desde el 2011 hasta el 2026 y que tiene como objetivos implementar un modelo de gestión que permita la participación de los ciudadanos, mejorar las condiciones de vida de éstos, entre otros. Es por estos antecedentes y tomando en consideración que las ciudades y regiones exitosas son aquellas que planifican su futuro, que radica la importancia de estudiar la metodología diseñada por el municipio de Portoviejo para evaluar las estrategias planificadas. Esta metodología debe permitir realizar el seguimiento y actualización de las estrategias a través de la valoración de los objetivos, informes de seguimiento, memorias anuales del plan, designación de agentes en el proceso de seguimiento y un cuadro de mando que permita finalmente evaluar el grado de cumplimiento de estas. En esta investigación se utilizó el método de búsqueda y revisión bibliográfica para normalizar los aspectos teóricos fundamentales sobre la planificación estratégica - evaluación y otros del nivel teórico (analítico - sintético, inductivo - deductivo). El método sistémico también permitió elaborar el proceso de evaluación y las relaciones entre sus etapas.

PALABRAS CLAVE: desarrollo local, Portoviejo, Plan de desarrollo, Evaluación, estrategias

1 Magister en Auditoría Integral; Blanca Victoria Cerruffo Briones, Universidad Técnica de Manabí. Emel:victoriacerruffo@yahoo.com

${ }^{2}$ Abg. Angela Marcelina Cerruffo Briones; Consejo de la Judicatura Ecuador

Emel: acerruffo@hotmail.com 
Blanca Victoria Cerruffo Briones, Angela Marcelina Cerruffo Briones, ...

\section{METHODOLOGY TO EVALUATE THE TERRITORIAL PLAN OF CANTÓN PORTOVIEJO}

\section{ABSTRACT}

The canton Portoviejo is a city in the province of Manabí belonging to the coastal region of Ecuador. The population according to the last population census is 280,029 inhabitants. In recent years has had a breakthrough in terms of urbanism, adequacy and infrastructure of basic services resulting from the planning developed by the municipality. But there is still a lot to do so that Portoviejo can provide the best living conditions for its inhabitants. For this purpose, the municipality designed the Territorial Development Plan that is in force from 2011 to 2026 and whose objectives are to implement a management model that allows citizens to participate and improve their living conditions, among others. It is for this background and taking into account that successful cities and regions are those that plan their future, which is the importance of studying the methodology designed by the municipality of Portoviejo to evaluate the planned strategies. This methodology should allow the monitoring and updating of the strategies through the assessment of the objectives, monitoring reports, annual reports of the plan, designation of agents in the monitoring process and a scorecard that finally allows the evaluation of the degree of compliance with them. In this research, the search and bibliographic review method was used to normalize the fundamental theoretical aspects of strategic planning - evaluation and others of the theoretical level (analytical - synthetic, inductive - deductive). The systemic method also allowed to elaborate the evaluation process and the relationships between its stages.

KEYWORDS: Local development, Portoviejo, Development plan, Evaluation, strategies

\section{INTRODUCCIÓN}

Antecedentes de la Planificación estratégica de ciudades

Las ciudades son el núcleo donde se asienta la sociedad humana desde la antigüedad y el ser humano en el transcurso de los años no ha escatimado en esfuerzos en hacer que estas permitan converger con las actividades comerciales, sociales, industriales que realiza el hombre. Desde sus inicios se puede observar que las primeras civilizaciones en cuanto a su infraestructura se refiere mostraron gran interés en utilizar diseños arquitectónicos que les permitan suplir con sus necesidades.

Pero no fue hasta finales del siglo XIX con la llegada de la revolución industrial que aparecieron las primeras estructuras urbanas modernas con problemáticas como el desarrollo sostenible, la tecnología, el desarrollo industrial y el impacto medio ambiental. Estos cambios a los que se enfrentaron las ciudades dieron el puntapié para que aparezcan los primeros modelos de desarrollo e instrumentos de planificación.

Posteriormente en el periodo de la II Guerra Mundial fue el punto más pleno de la planificación de ciudades para finalmente ser vista como un instrumento necesario para el desarrollo de las sociedades sobre todo para los capitalistas. Cabe recalcar que la teoría de planificación de ciudades empezó como iniciativa privada pero con el transcurso de los años fue tomando más un aspecto público a cargo de los municipios o ayuntamientos.

Continuando con los antecedentes en la década de los años 60 y teniendo en cuenta la mejora en la calidad de vida de las personas, esto provocó profundas transformaciones de tipo social, pues 
aumentó la demografía, se dinamizó la economía, el sector automotriz creció aceleradamente, así como el industrial trayendo consigo la contrapartida: el impacto negativo al medio ambiente.

En la década de los 80 (Güell, 2018) ocurre una crisis con respecto a la evolución de la planificación estratégica debido al crecimiento del modelo neroliberal en la economía de los países, el cual no aceptaba con buenos ojos la intervención de la parte gubernamental en los planes de desarrollo de ciudades. Y es así que en la últimas dos décadas aparece el nuevo orden económico teniendo como base la globalización, el boom del internet y el sector automotriz que ha logrado consigo el crecimiento de las ciudades pero cuyo crecimiento a largo plazo es insostenible.

Así mismo en la última década la preocupación por el medio ambiente y uso de centros urbanos compartidos hacen que nuevamente se toque el tema de la planificación de ciudades para hacer frente a los nuevos retos que afrontan y afrontarán las ciudades en el futuro. En el caso de Ecuador y específicamente Portoviejo, es recién en la última década que se ha implementado un modelo de planificación de ciudades adoptada por el municipio. Esta planificación obedece también al carácter impositivo del estado de obligar a los cantones a planificar para que sean desembolsados los rubros o partidas económicas para cada cantón.

\section{DESARROLLO}

Fundamentos de la planificación estratégica de ciudades

La planificación estratégica ha impactado en dos sectores de la sociedad como son el sector privado y posteriormente el sector público a continuación se realizará un resumen desde los dos sectores:

En el sector privado

La planificación estratégica nace a partir de las estrategias utilizadas en el campo militar basadas en principios como el de la economía de las fuerzas, seguridad, flexibilidad, maniobra. (Galdon, 1983). Posteriormente esa estrategia militar empezó a ser utilizada en el sector empresarial y luego en el sector público. En este ámbito la planificación estratégica adopta características que persiguen la obtención de lucro así como, la asignación de recursos con base presupuestaria, la identificación de los factores internos (debilidades fortalezas) y externos (amenazas y oportunidades).

Se da especial énfasis en el estudio del entorno sistémico de las empresas, es decir el análisis de los factores exógenos que puedan afectarla de forma negativa o positiva, presentes o futuros. En cuanto al entorno interno se deben identificar los factores claves como debilidades y puntos fuertes de la empresa, a través del análisis de ambos entornos se podrá determinar la posición que tiene la empresa frente a su competencia. La planificación estratégica de empresas tiene como precepto que una vez haya sido efectuado el estudio de sus componentes internos y externos de debe formular la visión estratégica y las diferentes estrategias plasmadas en el plan operativo con objetivos a corto plazo, por ultimo gradualmente se debe ir revisando estas estrategias y su cumplimiento para tomar decisiones asertivas.

En el sector público

Fue en la década de los años 70 y 80 donde se vio marcada la influencia de la planificación estratégica en este sector. Muchos de los gobiernos europeos y norteamericanos empezaron implementar las estrategias del sector privado en las organizaciones públicas con el fin de obtener la máxima rentabilidad de los recursos asignados a estas. 
Blanca Victoria Cerruffo Briones, Angela Marcelina Cerruffo Briones, ...

A diferencia de la metodología utilizada en el sector privado, Fernández Guell (2007) indica que se inicia con la negociación de un acuerdo donde participan todos los involucrados en el plan, se evalúan los mandatos o normativas externas que rigen a la entidad, se deben tener en cuenta la misión y valores de la organización, el diagnostico de los factores externo e internos de la empresa, muy similar al diagnóstico efectuado en el sector privado, se identifican y formulan las estrategias, se determina la visión estratégica y se implanta el modelo, finalizando con la comunicación a los interesados.

En este punto no menciona nada sobre la forma de evaluar el plan posteriormente, teniendo en cuenta lo beneficioso que podría ser para medir el cumplimiento de los objetivos planteado por la entidad pública.

En contra de la planificación estratégica

Así como esta temática tiene algunos seguidores, así mismo sus detractores, entre los argumentos que utilizan están los siguientes:

El futuro no se puede adivinar.

Un plan estratégico genera que aumente la burocracia y por ende el retraso del cumplimiento de la gestión gubernamental.

Representa un rubro muy costoso para las organizaciones.

Supone una camisa de fuerza para las instituciones del sector público.

Se enfoca en problemas a largo plazo y se despreocupa de los actuales.

Ante estas criterios la planificación ha logrado posicionarse en el sector privado como público, en el caso del Ecuador en la última década este concepto se encuentra normado en sus cuerpos jurídicos y dirigido por la Secretaria Nacional de Planificación.

Evaluación de planes estratégicos

Según Martín y Merinero (2010), es poco lo que se ha abordado acerca de la evaluación de los planes estratégicos, puesto que en su mayoría la bibliografía está enfocada en cómo realizar un plan y aplicarlo. Según Fernández (2007), en esta materia poco se ha avanzado y destaca la importancia de la evaluación puesto que sería como cosechar sin saber lo que se ha sembrado, ni cuando recoger la cosecha, destacando la importancia de la evaluación y revisión de los planes para el cumplimiento de las metas a corto y largo plazo:

Se percibía que se había avanzado poco en esta materia [referido a la evaluación], por lo cual se suscitaban ciertas cuestiones. ¿Cómo mantener vivos los Planes y conseguir que se obtengan resultados visibles a corto-medio plazo? ¿Cómo superar la fragmentación sectorial en la toma de decisiones? ¿Cómo desarrollar un proceso participativo eficaz durante la fase de implantación? (Fernández, 2007).

En el caso de la planificación de las ciudades este elemento de la revisión y evaluación permitirá a los cantones contar con una herramienta de medición de los impactos del plan y su repercusión en los sistemas de gobernanza y en cómo se desarrollan estos procesos para poder oportunamente corregir, ajustar los objetivos planteados en sus 4 niveles fin, propósito, componentes y actividades.

Así mismo Fernández (2007) destaca: 
No hay investigaciones recientes que valoren la efectividad de estos planes. [...] sería muy interesante determinar la capacidad real de la planificación estratégica para crear capital social, para integrar los diversos procesos de planificación sectoriales, para concertar las actuaciones de las Administraciones Públicas y para estimular la cooperación público-privada (Fernández, 2007: 636).

He aquí la importancia de abordar esta temática puesto que el plan estratégico se compone del diseño y ejecución de los procesos concernientes a la provisión de bienes colectivos y por ende de la toma de decisiones en caso de modificar los mismos. Pero no todo ha sido desesperanzador en torno a este temática, puesto que existen algunas experiencias de evaluación que abordan el tema de planificación de ciudades como las evaluaciones de los primeros planes estratégicos de Florencia (Perulli et al., 2008) y Venecia (Perulli et al., 2009), y no olvidarnos del primer plan estratégico de Turín (Dente y Melloni, 2005).

Desde esta perspectiva en el caso específico de la ciudad de Portoviejo, la Senplades (Secretaría nacional de planificación del Ecuador) en el Instructivo para ejecución de planes estratégicos ha establecido que cada ciudad deberá diseñar sus planes de evaluación y monitoreo a través de un modelo de gestión, del cual deberá trimestralmente presentar un informe a este organismo de control. De esta forma se pretende realizar la evaluación de los planes a nivel interno (municipio) y externo (organismo de control).

Metodología simplificada de la planificación estratégica de ciudades

Para el caso de estudio aplicaremos la metodología aplicada por Fernández (2007) como se demuestra a continuación:

\section{Gráfico No 1}

Metodología simplificada de la planificación estratégica de ciudades

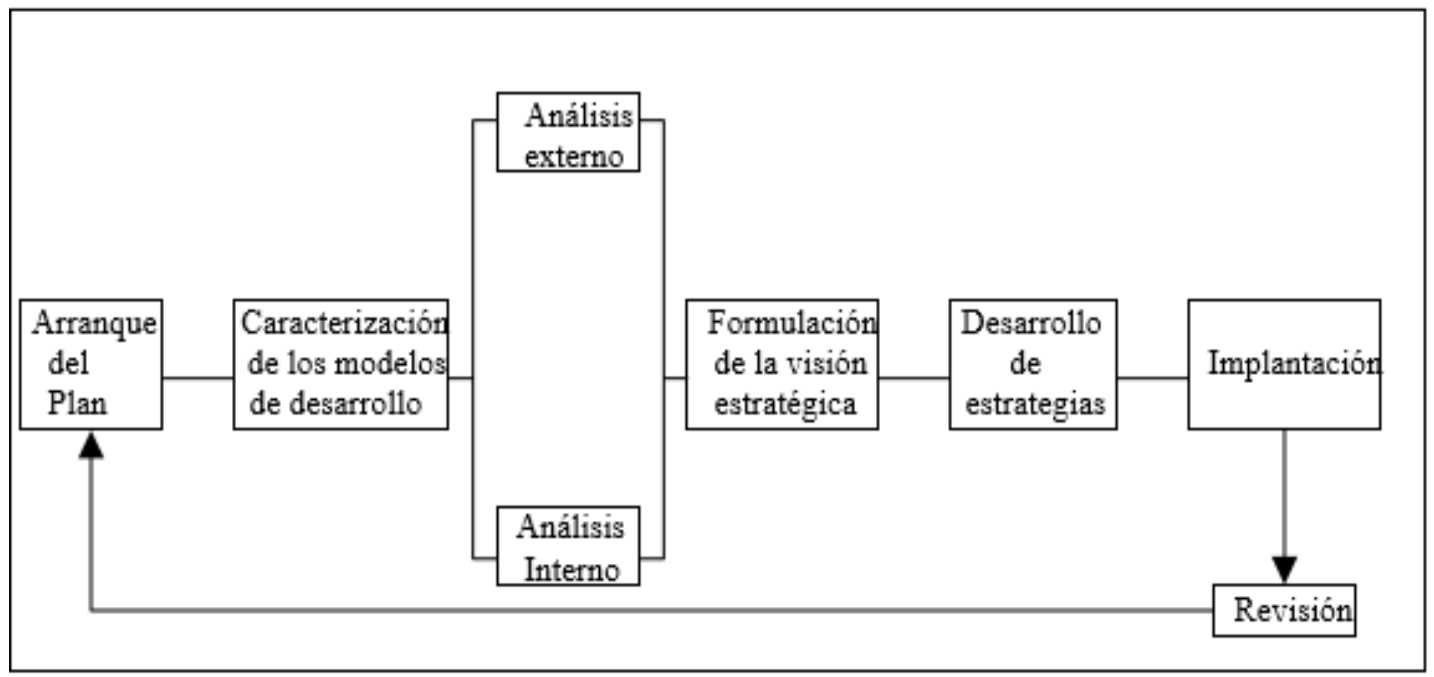

Fuente: Fernández Güell (1997).

Esta metodología utilizada por Fernández inicia con el diseño del plan donde se debe realizar una caracterización de los modelos de desarrollo a través del análisis externo e interno de la institución, aquí se utilizan herramientas de administración como el FODA, cuadro de mando integral, estudio sistémico de la organización entre otras. En el caso de estudio se debe realizar un diagnóstico por 
Blanca Victoria Cerruffo Briones, Angela Marcelina Cerruffo Briones, ...

componentes que permita identificar los problemas y potencialidades de la ciudad- municipio y un análisis estratégico territorial.

Con el estudio efectuado a través del diagnóstico se formula la visión estratégica, así como las estrategias específicas para llevar a cabo la consecución de la misma. El municipio de Portoviejo ha establecido de forma apropiada su visión, objetivos estratégicos de desarrollo y determinación de indicadores y metas, así como la definición de las categorías de ordenamiento territorial para la territorialización de políticas públicas. Posterior a este proceso sigue la implantación del plan para lo cual el Municipio de Portoviejo ejecuta las estrategias con enfoques a la articulación y coordinación para la gestión de los planes, metodologías de participación de la ciudadanía y las propuestas de programas con sus posibles fuentes de financiamiento, metas e indicadores, vinculados al Plan Nacional de Desarrollo. Por último, en la etapa de revisión propuesta por Fernández, el municipio ha elaborado un modelo de gestión que permite evaluar el plan y reportar al órgano de control el avance de este. En este punto es necesario abordar el uso de indicadores aplicados al plan propiamente, a la ciudad y al entorno. La evaluación de las estrategias no puede versar solo sobre el cumplimiento de estas, deberá revisarse también la repercusión en el impacto sobre la ciudad y en su entorno. Para el efecto se deberán utilizar indicadores de progreso del plan, indicadores de impacto del plan en la ciudad, e indicadores comparativos con otras ciudades que permitían medir el crecimiento del cantón con relación a otras.

Uso de indicadores

Entre los indicadores que permiten de forma más eficaz medir los planes estratégicos encontramos:

Gráfico No 2

Indicadores de uso común para medir planes estratégicos

\begin{tabular}{|cl|}
\hline Indicadores de Progreso & $\begin{array}{l}\bullet \text { eficiencia } \\
\bullet \text { eficacia }\end{array}$ \\
\hline Indicadores de Impacto & $\begin{array}{l}\bullet \text { mejoramiento de la salud } \\
\bullet \text { aceptacion del gobierno }\end{array}$ \\
\hline Indicadores de entorno & $\begin{array}{l}\bullet \text { población } \\
\bullet \text { salud }\end{array}$ \\
\hline Benchmarking de ciudades & \\
\hline Informes de progreso \\
\hline
\end{tabular}

Fuente: Fernández Güell. (2007). Planificación estratégica de ciudades.

Elaboración propia

Estos indicadores por si solos no representan instrumentos de medición a menos que el evaluador del plan los diseñe con el fin de responder si las estrategias se han diseñado en cumplimiento y función de la visión y misión de la organización, deben permitir realizar acciones de corrección que permitan mejorar en la marcha, entre otros.

\section{Materiales y métodos}


En esta investigación se utilizó el método de búsqueda y revisión bibliográfica para normalizar los aspectos teóricos fundamentales sobre la planificación estratégica-evaluación y otros del nivel teórico (analítico, deductivo). El método deductivo que parte de las teorías generales de la planificación para centrarse en el caso específico de la ciudad de Portoviejo, mediante el razonamiento lógico para poder sustentar conclusiones finales. Este proceso parte de los análisis antes planteados, fundamentos, leyes y principios validados para ser aplicados a nuestro caso en particular, partiendo de una premisa concluir el fenómeno objeto de estudio e implementar las soluciones. Se utilizó el plan de desarrollo de la ciudad de Portoviejo, así como los instructivos para su elaboración.

\section{CONCLUSIONES}

El municipio de Portoviejo ha adoptado el modelo de evaluación de planes estratégicos propuesto por la Senplades, que consta de 4 etapas, las mismas que tiene apego a lo establecido por diferentes autores sobre todo a la metodología establecida por Fernández Guell. El uso de indicadores resulta imprescindible para la evaluación de los objetivos estratégicos, los mismos que deberán ser aplicados para medir los objetivos en todos sus niveles. Así mismo es importante que se determine a los responsables del seguimiento y monitoreo del plan, los cuales deberán verificar periódicamente y de forma sistemática el cumplimiento de cada objetivo constatando con los medios de verificación establecidos por el cabildo. Es importante que los municipios consideren que las ciudades y regiones exitosas son aquellas que planifican su futuro, en el caso de Portoviejo, es en la última década que se ha hecho énfasis a la planificación estratégica de la ciudad, en cuanto a la metodología aplicada para medir sus planes de desarrollo estas les permiten realizar el seguimiento y actualización de las mismas a través de la valoración de los objetivos, informes de seguimiento, memorias anuales del plan, designación de agentes en el proceso de seguimiento y un cuadro de mando que permita finalmente evaluar el grado de cumplimiento de estas.

\section{REFERÉNCIAS BIBLIOGRÁFICAS}

Constitución de la República del Ecuador (2008). Ciudad Alfaro: Asamblea Constituyente

COOTAD (Código Orgánico de Organización Territorial, Autonomía y Descentralización) (2010). Quito: Asamblea Nacional.

COPFP (Código Orgánico de Planificación y Finanzas Públicas) (2010). Quito: Asamblea Nacional.

Fernández Güell, J. M. (2007). 25 años de planificación estratégica de ciudades, Ciudad y Territorio.

Fernández Güell, J. M. (2007). Planificación estratégica de ciudades. Barcelona: Editorial Reverté S. A.

Galdón Domenech, Domingo. (1983). Introducción a la estrategia. Madrid: Escuela Superior del Aire, 1983.

INEC (Instituto Nacional de Estadística y Censos) (2010a). Censo de población y vivienda. Quito: INEC.

Martín Mesa, A., y Merinero Rodríguez, R. (dirs.) (2010): Planificación estratégica territorial. Estudios metodológicos. Sevilla: Junta de Andalucía, Consejería de Gobernación y Justicia.

Perulli, P. (2007). Griglia metodologica per l'inquadramento dei piani strategici, en T. Pugliese (ed.) Monitoraggio e valutazione dei piani strategici. Firenze: Rete delle Città Strategiche (ReCS)

Secretaría Nacional de Planificación y Desarrollo - Senplades. (2013). Plan Nacional de Desarrollo / Plan Nacional para el Buen Vivir 2013-2017. Quito, Ecuador

Secretaría Nacional de Planificación y Desarrollo - Senplades. (2017). Plan Nacional de Desarrollo 2017-2021, Toda una Vida. Quito - Ecuador. 
Blanca Victoria Cerruffo Briones, Angela Marcelina Cerruffo Briones, ...

34 UNESUM-Ciencias. Publicación cuatrimestral. Vol. 3, Año 2019, No. 2 (Mayo - Agosto) 